Special issue of the 2nd International Conference on Computational and Experimental Science and Engineering (ICCESEN 2015)

\title{
Effects of Coherent Synchrotron Radiation in Bunch Compressor of TARLA
}

\author{
H. YILDIZ ${ }^{a, *}$, A. AKSOY ${ }^{b}$ AND P. ARIKAN ${ }^{a}$ \\ ${ }^{a}$ Gazi University, Physics Department, Ankara, Turkey \\ ${ }^{b}$ Ankara University, Institute of Accelerator Technologies, Ankara, Turkey
}

\begin{abstract}
High order and non-linear effects in accelerator beamline play an important role in the transport of the beam. These effects cause variation of designed beam parameters such as emittance growth, bunch length variation, beam halo formation, etc. One of the known non-linear effects in bunch compressors is the coherent synchrotron radiation effect. In this study we focus on coherent synchrotron radiation effect in Turkish Accelerator and Radiation Laboratory in Ankara (TARLA) machine which is designed to drive Infrared Free Electron Laser, covering the range of 3-250 $\mu \mathrm{m}$. We additionally discuss high order effects in bunch compressor of TARLA.
\end{abstract}

DOI: 10.12693/APhysPolA.130.214

PACS/topics: 29.27.Bd, 29.27.-a, 29.20.Ej, 41.60.-m, 41.60.Cr, 41.85.Ja, 41.85.-p, 07.55.Db, 02.10.Yn

\section{Introduction}

The Turkish Accelerator and Radiation Laboratory in Ankara (TARLA) is proposed to generate Infrared Free Electron Lasers (IR-FEL) in oscillator mode, covering the range between 3 and $250 \mu \mathrm{m}$, using two optical resonator systems. The machine will mainly consists of an injector based on normal conducting technology, two superconducting accelerating modules separated by a bunch compressor, bremsstrahlung line and FEL generation system [1]. The schematic view of the facility is given in Fig. 1 and the main electron beam parameters of TARLA are given in Table I [2].

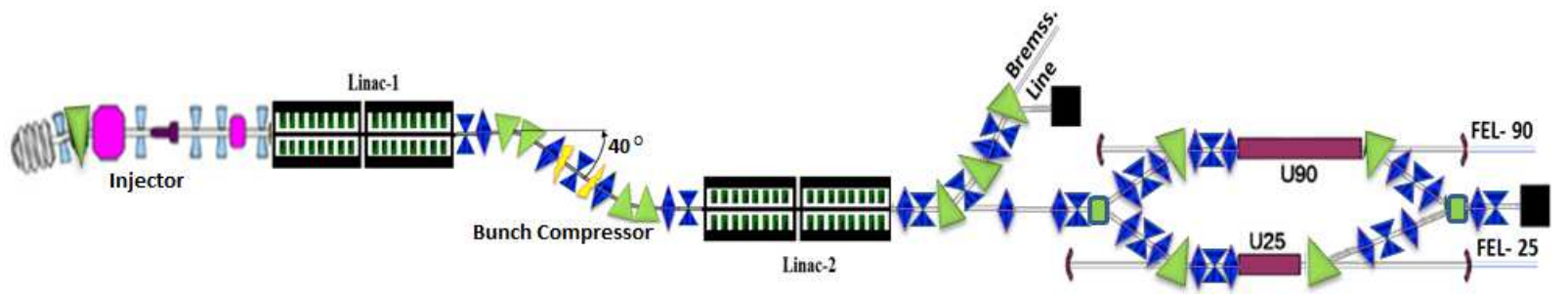

Fig. 1. Schematic layout of TARLA. Green shapes indicate location of dipoles, blue shapes are quadrupole magnets, cyan symbols are solenoid magnets, magenta shapes are buncher cavities and maroon shapes represent undulator magnets.

Since generation of FEL requires high peak current with sufficiently small bunch length, especially for shorter wavelengths, TARLA beamline has been designed for minimum bunch length at maximum beam energy. To do that a dog-leg type of bunch compressor has been designed. The main goal of this study is calculation of coherent synchrotron radiation (CSR) and space charge effects in the bunch compressor section, in order to improve the current bunch compressor design of TARLA. Usually before the bunch compressor section, the energy spread is purposely increased by RF cavity to have a large compression factor. After the bunch compression process, large energy spread can be reduced by the cavities located after the bunch compressor for the latest applications. However, large energy spread may make the transport of the electron bunch through the bunch

*corresponding author; e-mail: huseyinyildiz006@gmail.com

\section{TABLE I}

Electron beam parameters of TARLA.

\begin{tabular}{c|c|c}
\hline \hline Parameter & Base & Unit \\
\hline Beam energy & $15-40$ & $\mathrm{MeV}$ \\
Max average beam current & 1 & $\mathrm{~mA}$ \\
Max bunch charge & 77 & $\mathrm{pC}$ \\
Horizontal emittance & $<15$ & $\mathrm{~mm} \mathrm{mrad}$ \\
Vertical emittance & $<15$ & $\mathrm{~mm} \mathrm{mrad}$ \\
Longitudinal emittance & $<40$ & $\mathrm{keV} \mathrm{ps}$ \\
Bunch length & $0.3-6$ & $\mathrm{ps}$ \\
Bunch repetition frequency & 13 & $\mathrm{MHz}$
\end{tabular}

compressor more difficult, due to dispersive and chromatic effects. We have re-optimized the required energy spread, taking into account the effects of space charge forces and CSR. Parmela [3] and Elegant [4] codes have been used for tracking the beam. Parmela has been used for tracking from gun to end of the bunch compressor, 
in order to investigate space charge effect on the bunch. Elegant has been used for tracking from the exit of the first cavity of linac-1 to the end of the bunch compressor, in order to check the influence of CSR and space charge on the bunch.

\section{Bunch compression}

The bunches that have a proper relative energy spread can be compressed by passing bunch through a dispersive region that is generated by bending magnets. The lattice that creates dispersive regions usually can be chicane or dogleg plus multipoles, depending on the sign of the energy chirp. For a bunch with lower energy at the tail and higher energy at head like at TARLA, one should choose positive $R_{56}$, which is the dogleg type of bunch compressors. The path length difference of bunch compressor for particles with a relative energy deviation $\delta$ is given by $[5,6]$

$$
\Delta z=z_{2}-z_{1}=\eta \delta=R_{56} \delta+T_{566} \delta^{2}+U_{5666} \delta^{3}+\ldots
$$

where $\eta$ is longitudinal dispersion, $z_{1}$ is uncompressed bunch length, $z_{2}$ is the compressed bunch length, $R_{56}$ is linear longitudinal dispersion, $T_{566}$ and $U_{5666}$ are second and third order longitudinal dispersion respectively.

\subsection{Bunch compression in TARLA}

The capture process from the injector into the first superconducting cavity of linac-1 [2], results in bunches having opposite chirp (lower energy at the tail, higher energy at the head). One could use second cavity of linac-1 off-crest to change sign of chirp of bunches and to use chicane type of bunch compressor. However that would cause large energy loss for short bunch operation [7]. In order to operate all cavities on-crest a dogleg type bunch compressor has been designed. To have a large longitudinal dispersion, two couples of dipole magnets have been used. The pair of the dipoles is separated by a quadrupole triplet to change the direction of the dispersion and achromaticity. All dipoles used in the bunch

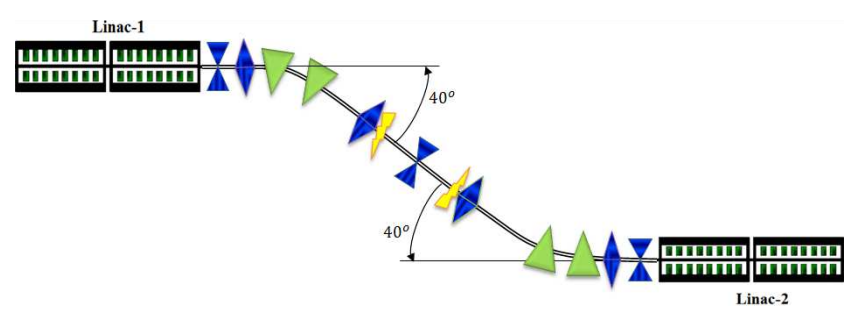

Fig. 2. Layout of the bunch compressor of TARLA. Green shapes are dipole magnets, blue shapes are quadrupole magnets and yellow symbols are sextupole magnets.

compressor, were chosen to be the same rectangular magnets with bending angle of 20 degrees, entrance and exit angle of 5 degrees and length of $0.15 \mathrm{~m}$. In addition, quadrupole doublets have been used to match the electron bunch at the entrance and exit of the compressor as shown in Fig. 2

\subsection{CSR effect in bunch compressor of TARLA}

When a bunch passes through a dipole magnet, it emits radiation [8]. If wavelength of the emitted radiation is longer than the bunch length $\left(\lambda_{r}>\sigma_{z}\right)$ the radiation is called CSR [9]. It is known that the CSR leads to growth of the emittance and of the energy spread within the bunch [10]. In other words the CSR forces particles to follow different paths than the ideal one, due to energy loss. Thus the particles have position and angle offsets after they leave the dipole. In order to estimate the influence of CSR on the bunch we have used the Elegant code, using the bunch distribution created by Parmela at the exit of the first linac. It has been shown in Ref. [2] that the CSR causes $14.5 \%$ and $5 \%$ growth of the horizontal emittance and of the bunch length, respectively. In order to reduce the impact of the CSR and of the second order effects we propose a modification of the bunch compressor. The sextupoles have been successfully used in a bunch compressor to correct the nonlinearities and second order effects in the phase space transformation of charged particles.

The second order term $T_{566}$ is created by dipoles and it is also amplified by the quadrupole magnets. The dispersive effects of these magnets can be calculated using their matrix representations. After multiplying the matrices, term $T_{566}$ may be expressed as follows [6]

$$
T_{566}=4 \rho \sin ^{2}(\theta / 2) \cos (\theta / 2)+\sum_{w} a_{i 6 k} T_{i 6 k},
$$

where $\rho$ is the bend radius of the dipole, $\theta$ is the bend angle of the dipole, and $a_{i 6 k}$ are coefficients, which are functions of $\rho$ and $\theta$. The summation is over the set $w$ of values of $i, k$ corresponding to the nonzero transverse second-order chromatic terms. If we want to use two sextupole magnets, having field strengths $\kappa$ and $\alpha \kappa$ respectively, then Eq. (2) can be obtained as [6]

$$
T_{566}=A-C(1-\alpha) \kappa .
$$

Here quantities $A$ and $C$ are functions of matrix elements that comprise $\rho, \theta$, drift lengths and focal lengths of the quadrupoles. And $\alpha$ represents the ratio of the two sextupoles' field strengths. There is linear dependence on $\kappa$ because the second-order matrix elements for a sextupole are proportional to the field strength. Since the nonlinear horizontal chromaticity of the focusing quadrupoles amplifies the second-order longitudinal dispersion $T_{566}$ and then it grows linearly in the drift section after each quadrupole. For that the sextupole magnets should be located close to the horizontally focusing quadrupoles [11]. The sextupole magnets, which are $5 \mathrm{~cm}$ long, are placed close to outer quadrupoles, as shown in Fig. $2(\alpha=-1$, because the sextupole fields are equal in magnitude, but have opposite polarity). Thus yields second order term as,

$$
T_{566}=0.244 m-\left(0.000564 m^{4}\right) \kappa .
$$

As it can be seen, in case of absence of the sextupoles, the $T_{566}$ has a value of $0.244 m$. But it can be forced to zero at a sextupole field strength of $\kappa=420 \mathrm{~m}^{-3}$. This means reduction of second order effects in the bunch compressor is possible analytically. 

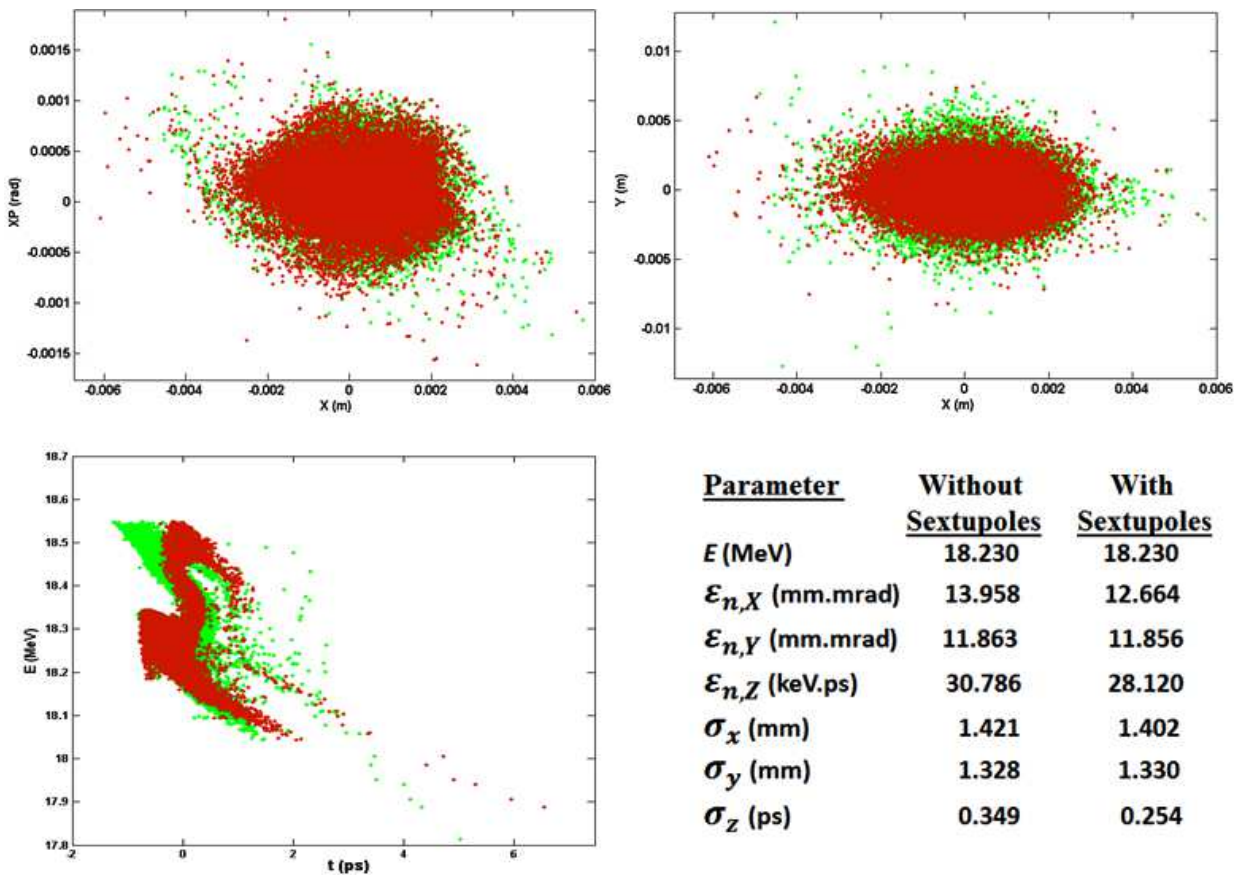

\begin{tabular}{|c|c|c|}
\hline$\underline{\text { Parameter }}$ & $\begin{array}{l}\text { Without } \\
\text { Sextupoles }\end{array}$ & $\begin{array}{c}\text { With } \\
\text { Sextupoles }\end{array}$ \\
\hline$E(\mathrm{MeV})$ & 18.230 & 18.230 \\
\hline$\varepsilon_{n, X}$ (mm.mrad) & 13.958 & 12.664 \\
\hline$\varepsilon_{n, Y}$ (mm.mrad) & 11.863 & 11.856 \\
\hline$\varepsilon_{n, Z}$ (keV.ps) & 30.786 & 28.120 \\
\hline$\sigma_{x}(\mathrm{~mm})$ & 1.421 & 1.402 \\
\hline$\sigma_{y}(\mathrm{~mm})$ & 1.328 & 1.330 \\
\hline$\sigma_{z}(\mathrm{ps})$ & 0.349 & 0.254 \\
\hline
\end{tabular}

Fig. 3. Transverse beam distribution (topright), and horizontal (topleft) and longitudinal (bottomleft) phase spaces and beam parameters of the bunch after the bunch compressor with sextupoles (reddots) and without sextupoles (greendots).

Using the analytical results we have performed simulation using Elegant code and beam parameters given in Table I. Figure 3 shows the phase space plots of the bunch after the bunch compressor, with and without sextupole magnets. As it can be seen from the figure, there is a relatively high reduction of horizontal emittance growth and the longitudinal charge distribution is improved.

\section{Conclusions}

The beamline of TARLA has been optimized for minimum bunch length at maximum beam energy, which is very important case of low wavelength FEL operation. To reduce the CSR and the second order effects in bunch compressor we propose to use sextupole magnets. Re-optimization of the beamline has been carrie out. At first stage of optimization we have used analytical expressions. At second stage we have used well know simulation tools. After the optimization, the bunch length is reduced from 349 fs to 254 fs (27.5\% reduction), the horizontal emittance is about $12.664 \mathrm{~mm} \mathrm{mrad}$ (i.e. decreased by $9.27 \%$ ), and longitudinal emittance is also decreased. Since the performance of FEL strongly depends on peak beam current, we expect about $30 \%$ enhancement of its gain. In addition weak field effects, misalignment, magnet errors and longitudinal errors will affect beam transportation. These effects should also be studied.

\section{Acknowledgments}

This work was supported by the Scientific and Technological Research Council of Turkey (TUBITAK, BIDEB-
2211-C) and Turkish Republic Ministry of Development (TRMOD) with Grant No: DPT2006K-120470.

\section{References}

[1] A. Aksoy, O. Karslı, The Technical Design Report of TARLA IAT (2015).

[2] A. Aksoy, U. Lehnert, Nucl. Instrum. Meth. A 762, 54 (2014).

[3] L.M. Young, Parmela User Manual, Report No. LAUR-96-1835, Los Alamos National Laboratory, Revised April 2003, (1996).

[4] M. Borland, User's Manual for Elegant, Program Version 28.1.0, Advanced Photon Source (2015).

[5] M. Dohlus, T. Limberg, P. Emma, ICFA Beam Dynamics Newsletter 38, 15 (2005).

[6] R.J. England, J.B. Rosenzweig, G. Andonian, P. Musumeci, G. Travish, R. Yoder, Phys. Rev. ST Accel. Beams 8, 012801 (2005).

[7] U. Lehnert, A. Aksoy, M. Helm, P. Michel, H. Schneider, W. Seidel, S. Winnerl, Proceedings of FEL Conference 2010, Malmo, Sweden, JACoW, 2010, p. 656.

[8] J.D. Jackson, Classical electrodynamics, 3rd ed., Wiley, New York 1998.

[9] T. Nakazato, M. Oyamada, N. Niimura, S. Urasawa, R. Kato, Y. Shibata, K. Ishi, T. Ohsaka, T. Takahashi, H. Mishiro, M. Ikezawa, Y. Kondo, T. Nanba, Y. Torizuka, Part. Accel. 33, 141 (1990).

[10] M. Borland, Phys. Rev. ST Accel. Beams 4, 070701 (2001).

[11] A.K. Çiftçi, R. Çiftçi, H. Yıldız, K. Zengin, Nucl. Instrum. Meth. A 675, 34 (2012). 